\title{
Parasitosis intestinal en preescolares y escolares atendidos en el centro médico EsSalud de Celendín, Cajamarca
}

\author{
Jimmy Rinaldo Morales Del Pino ${ }^{1,2}$
}

\section{RESUMEN}

Objetivo: Determinar la prevalencia de parasitosis en niños en edad preescolar y escolar del distrito de Celendín, atendidos en el centro médico EsSalud y encontrar presencia de los subtipos parasitarios.

Material y métodos: Estudio observacional, descriptivo y tipo transversal, realizado entre julio del 2015 y enero del 2016 en el servicio de laboratorio clínico del centro médico EsSalud de Celendín, Cajamarca. Se usó una ficha de recolección de datos por participante para recopilar datos clínicos, datos sociodemográficos e interrelación personal. Se procesaron muestras seriadas parasitológicas de 96 niños usando examen directo, test de Graham y la técnica de sedimentación espontánea. Se empleó un análisis univariado para encontrar frecuencias, porcentajes y desviaciones estándar, y para el análisis bivariado se empleó la prueba de Chi-cuadrado y el test exacto de Fisher para asociar el grado de parasitismo con cada variable obtenida.

Resultados: La prevalencia fue $90.6 \%$ (87/96). Se encontró Blastocystis hominis $81.2 \%$, lodamoeba butschlii $6.3 \%$, Endolimax nana 19.8\%, Entamoeba coli 35.4\%, Chilomastix mesnilii 13.5\%, Giardia lamblia 9.4\%, Enterobius vermicularis $16.7 \%$ y Ascaris lumbricoides $1.0 \%$. Un 20.8\% (20/96) de los participantes presentaron estructuras semejantes a Urbanorum spp. Predominó el multiparasitismo $60.4 \%$ y hubo asociación estadísticamente significativa entre el nivel de educación y el grado parasitario $(\mathrm{p}=0.017)$.

Conclusiones: Hubo alta prevalencia de parasitosis en niños en edad preescolar y escolar del distrito de Celendín atendidos en el centro médico EsSalud, siendo Blastocystis hominis el parásito con más predominio.

Palabras clave: parásitos, protozoarios, helmintos, niños, nuevo parásito.

\section{Intestinal parasitism in preschool and school students treated in the EsSalud Medical Center of Celendín, Cajamarca}

\section{ABSTRACT}

Objective: To determine the prevalence of parasitic infections in preschool and school children of Celendín district, treated at the EsSalud medical center and find the presence of parasitic subtypes.

Material and methods: Observational, descriptive, cross-sectional research, conducted between July 2015 and January 2016 in the clinical laboratory service of EsSalud Medical Center of Celendín, Cajamarca. A data collection sheet was used for each participant to collect clinical data, demographic data and personal interaction.

Parasitological samples of 96 children were processed by using direct examination, test of Graham and spontaneous sedimentation technique. Univariate analysis was used to find frequencies, percentages and standard deviations, and for the bivariate analysis we used Chi-square and Fisher's exact tests to associate the degree of parasitism with each variable obtained.

Results: The prevalence was $90.6 \%$ (87/96). Blastocystis hominis $81.2 \%$, iodamoeba bütschlii $6.3 \%$, Endolimax nana $19.8 \%$, Entamoeba coli $35.4 \%$, Chilomastix mesnilii 13.5\%, Giardia lamblia 9.4\%, Enterobius vermicularis $16.7 \%$ was found and Ascaris lumbricoides 1.0\%. 20.8\% (20/96) of the participants had similar structures to Urbanorum spp. Multiparasitism predominated $(60.4 \%)$ and there was a statistically significant association between the level of education and parasitical degree $(p=0.017)$.

Conclusions: There was a high prevalence of parasitic infections in children in preschool and school age of Celendín district attended at the EsSalud medical center being Blastocystis hominis parasite the most predominant.

Key words: parasites, protozoa, helminths, children, new parasite.

1. Bachiller en Tecnología Médica, área de Laboratorio Clínico y Anatomía Patológica.

2. Centro Médico EsSalud Celendín. Servicio de Laboratorio Clínico. 


\section{INTRODUCCIÓN}

El parasitismo está considerado como un problema de salud pública a nivel mundial, estimándose en más de un cuarto de la población del mundo con parásitos, siendo la población infantil la más afectada. Existen muchas causas que originan parasitismo tales como la condición socioeconómica, el déficit en la higiene tanto personal como comunitaria, etc ${ }^{(1-9)}$.

La población que se encuentra en edad preescolar y escolar, también están propensos a ser parasitados, ocasionándoles una serie de síntomas que pueden, incluso conllevarlos a la muerte ${ }^{(2,5,9,10-14)}$.

En el Perú, país en vía de crecimiento, se ha podido encontrar una prevalencia alta, siendo del $64 \%$ para los parásitos de tipo patógeno, lo cual demuestra que nuestro país no es ajeno a esta enfermedad. También se ha observado que 1 de cada 3 peruanos se halla infectado con 1 o más tipos de parásitos y que según la región, predomina un tipo diferente, tal es así que los protozoarios abundan en la costa y sierra, mientras que los helmintos están más presentes en la selva ${ }^{(5,11,15,16)}$.

En el departamento de Cajamarca, sierra norte del Perú, diversos estudios han encontrado altas tasas de parasitosis. Rúa y col. encontró en la zona de Llama, un $80.7 \%$ de prevalencia en escolares y Cholán y col. halló en el $100 \%$ de niños muestreados en la ciudad de Cajamarca la presencia de parásitos ${ }^{(14,17-20)}$.

Si bien es cierto que Cajamarca ha sido muy estudiado en cuanto al reporte de tasas parasitarias, su zona este que comprende la provincia de Celendín, cercana a la selva, no presenta aún estudio alguno. Es por ello que el objetivo del presente trabajo es determinar la prevalencia de parasitosis en niños en edad preescolar y escolar del distrito de Celendín que fueron atendidos en el centro médico EsSalud así como también encontrar presencia de subtipos parasitarios.

\section{MATERIAL Y MÉTODOS}

Estudio de carácter observacional, descriptivo y tipo transversal, realizado entre julio del 2015 y enero del 2016 en el servicio de laboratorio clínico del centro médico EsSalud de Celendín, Cajamarca. Las muestras se obtuvieron de forma no probabilística, considerando a niños entre el rango de edades de 3 a 14 años y que estuvieran en el nivel preescolar y escolar, obteniendo una población de 130 niños.
Se excluyeron muestras contaminadas con orina, muestras no seriadas, niños que no trajeron muestras y aquellos con tratamiento antiparasitario actual.

Previo a la recolección de muestras, se informó a cada padre de familia el objetivo del estudio y se les entregó el consentimiento informado para que lo firmen y autoricen participar a su hijo. Así mismo se les entregó tres frascos para heces y dos láminas portaobjetos para el test de Graham, explicándoles la manera adecuada de recolectar la muestra. Se usó una ficha de recolección de datos por participante, donde se recopilaron datos clínicos, datos sociodemográficos e interrelación personal.

Las muestras de heces fueron recolectadas de manera seriada conjuntamente con las láminas para detectar oxiuros. Para el examen microscópico se utilizó el examen directo con lugol parasitológico y suero fisiológico $0,08 \%$. Las láminas del test de Graham fueron leídas inmediatamente. Se usó la técnica de sedimentación espontánea, la cual no requiere centrifugación y que permite detectar quistes, trofozoitos de protozoarios, huevos y larvas de helmintos ${ }^{(21)}$.

Para el análisis de datos, todos los resultados fueron ingresados a una hoja de cálculo de Microsoft Excel 2013, luego fueron analizados con el paquete estadístico IBM SPSS-22 Statistics. En el análisis univariado se calculó frecuencias y porcentajes para variables discretas y medias con desviaciones estándar para variables continuas. El análisis bivariado buscó asociaciones entre el grado de parasitismo con los datos sociodemográficos e interrelación personal, utilizándose la prueba de Chi-cuadrado y la prueba exacta de Fisher. Se consideró $\mathrm{p}<0.05$ como nivel de significación estadística.

\section{RESULTADOS}

Se procesaron muestras seriadas de 96 niños (34 fueron excluidos por los criterios mencionados anteriormente), donde el $45.8 \%$ fue del sexo masculino y el $54.2 \%$ del sexo femenino. La edad media fue de $7.97 \pm 3.19$ años con una edad mínima de 3 años y una máxima de 14 . El $37.5 \%$ estuvieron entre 3 y 6 años así como entre 7 y 10 años, mientras que el $25 \%$ estuvo entre 11 y 14 años (Tabla 1 ). 
Tabla 1. Rango de edades obtenidas de niños en edad preescolar y escolar

\begin{tabular}{lcc} 
Rango de edades & $\mathrm{n}$ & $\%$ \\
\hline 3-6 años & 36 & 37,5 \\
7-10 años & 36 & 37,5 \\
11-14 años & 24 & 25,0 \\
\hline Total & 96 & 100,0
\end{tabular}

En cuanto al nivel de educación, 31.3\% correspondió al nivel preescolar (jardín) y el $68.7 \%$ estuvo en el nivel escolar $49 \%$ en primaria y $19.7 \%$ en secundaria. El número promedio de hijos fue $2.31 \pm 1.009$ por familia, siendo el rango entre 1 a 6 hijos.

La prevalencia global encontrada en el estudio de muestras parasitológicas positivas fue de $90.6 \%$
$(87 / 96)$. Los tipos de parásitos hallados por nivel de educación se muestran en la tabla 2, correspondiendo a Blastocystis hominis $81.2 \%$ y Enterobius vermicularis $16.7 \%$ como los parásitos de mayor prevalencia para los de tipo no patógeno y patógeno, respectivamente.

Tabla 2. Frecuencia de parásitos intestinales por nivel de educación

\begin{tabular}{lrrrrrr} 
& \multicolumn{3}{c}{ Nivel de educación } \\
Tipos de parásitos & Pre escolar & Escolar & Total \\
\cline { 2 - 6 } & $\mathrm{n}$ & $\%$ & $\mathrm{n}$ & $\%$ & $\mathrm{n}$ & $\%$ \\
\hline Parásitos no patógenos & & & & & & \\
Blastocystis hominis & 27 & 28.1 & 51 & 53.1 & 78 & 81.2 \\
lodamoeba butschlii & 0 & 0.0 & 6 & 6.3 & 6 & 6.3 \\
Endolimax nana & 5 & 5.2 & 14 & 14.6 & 19 & 19.8 \\
Entamoeba coli & 8 & 8.3 & 26 & 27.1 & 34 & 35.4 \\
Chilomastix mesnili & 4 & 4.2 & 9 & 9.3 & 13 & 13.5 \\
Parásitos patógenos & & & & & 13 & 16.4 \\
Giardia lamblia & 6 & 6.3 & 3 & 3.1 & 9 & 9.4 \\
Enterobius vermicularis & 5 & 5.2 & 11 & 11.5 & 16 & 16.7 \\
Ascaris lumbricoides & 0 & 0.0 & 1 & 1.0 & 1 & 1.0 \\
\hline
\end{tabular}

Según el grado de parasitismo (Tabla 3) se encontró un $30.2 \%$ con monoparasitismo y para más de una forma parasitaria por paciente (multiparasitismo) un
$60.4 \%$, que abarcó biparasitismo $31.3 \%$, triparasitismo $26.0 \%$ y tetraparasitismo $3.1 \%$.

Tabla 3. Grado de parasitismo obtenido en niños en edad preescolar y escolar

\begin{tabular}{|c|c|c|c|}
\hline \multirow{2}{*}{\multicolumn{2}{|c|}{ Grado de parasitismo }} & \multicolumn{2}{|c|}{ Total } \\
\hline & & $\mathrm{n}$ & $\%$ \\
\hline \multirow[t]{2}{*}{ Monoparasitismo } & & 29 & 30.2 \\
\hline & Biparasitismo & 30 & 31.3 \\
\hline \multirow[t]{2}{*}{ Multiparasitismo } & Triparasitismo & 25 & 26.0 \\
\hline & Tetraparasitismo & 3 & 3.1 \\
\hline \multicolumn{2}{|l|}{ Negativo } & 9 & 9.4 \\
\hline \multicolumn{2}{|l|}{ Total } & 96 & 100 \\
\hline
\end{tabular}


En relación a los datos sociodemográficos e interrelación personal, recopilados por la ficha de recolección, se obtuvo que un $85.4 \%$ de los niños procedían de la zona urbana y un $14.6 \%$ de la zona rural; un $97.9 \%$ tuvo lactancia materna exclusiva y un $2.1 \%$ no. Un $89.6 \%$ consumía agua potable y el resto $10.4 \%$ no potable. También se encontró que el $87.5 \%$ de los niños usaban calzado y el $12.5 \%$ andaban descalzos; el $88.5 \%$ de los niños se lavaba las manos y el $11.5 \%$ no lo hacía; El 93.8\% contaban con desagüe y el $6.2 \%$ no tenían desagüe; el $70.8 \%$ consumía ensaladas y el $29.2 \%$ no consumía.

En cuanto al hacinamiento (definido cuando más de tres personas comparten una habitación), un 37.5\% tenían hacinamiento y el $62.5 \%$ no lo presentaban. Para la eliminación de excretas, un $93.8 \%$ tenían baño y el $6.2 \%$ letrina. El piso de casa era para un $35.4 \%$ de tierra y para un $64.6 \%$ de cemento; el $57.3 \%$ de los niños tenían animales domésticos en casa y el $42.7 \%$ no tenían.
Finalmente el $31.3 \%$ recibió último tratamiento entre los 3 a 6 meses, el $57.3 \%$ lo recibió hace más de 6 meses y un 11.5\% nunca habían recibido tratamiento.

Durante la evaluación clínica médica se encontraron síntomas como: dolor abdominal $14.6 \%$, diarrea $3.1 \%$, vómitos $4.2 \%$, estreñimiento $3.1 \%$, prurito anal $2.1 \%$, inapetencia $8.3 \%$, cefalea $4.2 \%$, fiebre $5.2 \%$, rinorrea $6.3 \%$, tos $11.5 \%$ y otros síntomas $22.9 \%$.

Al realizarse el análisis bivariado entre los factores sociodemográficos e interrelación personal con el grado de parasitismo, únicamente se obtuvo asociación con el nivel de educación ( $p=0.017)$, es decir, la cantidad de formas parasitarias (multiparasitismo) era mayor en aquellos que estaban en el nivel escolar. El resto de factores (Tabla 4) no tuvieron asociación estadísticamente significativa $(p>0.05)$

Tabla 4. Análisis bivariado entre factores sociodemográficos e interrelación personal con el grado de parasitismo

\begin{tabular}{|c|c|c|c|}
\hline \multirow[b]{2}{*}{ Factores } & \multicolumn{2}{|c|}{ Grado de parasitismo } & \multirow[b]{2}{*}{ Valor $\mathrm{p}$} \\
\hline & monoparasitismo & multiparasitismo & \\
\hline \multicolumn{4}{|c|}{ Procedencia } \\
\hline Urbana & $27(93.1)$ & $47(81.0)$ & $0.204(a)$ \\
\hline Rural & $2(6.9)$ & $11(19.0)$ & \\
\hline \multicolumn{4}{|c|}{ Nivel de educación } \\
\hline Pre escolar & $15(51.7)$ & $15(25.9)$ & $0.017 \quad(\mu)$ \\
\hline Escolar & $14(48.3)$ & $43(74.1)$ & \\
\hline \multicolumn{4}{|c|}{ Lactancia exclusiva } \\
\hline Sí & $28(96.6)$ & $58(100.0)$ & $0.333(a)$ \\
\hline No & $1(3.4)$ & $0(0.0)$ & \\
\hline \multicolumn{4}{|c|}{ Tipo de agua } \\
\hline Potable & $24(82.8)$ & $53(91.4)$ & $0.291(a)$ \\
\hline No potable & $5(17.2)$ & $5(8.6)$ & \\
\hline \multicolumn{4}{|c|}{ Número de hijos } \\
\hline $1-3$ & $28(96.6)$ & $53(91.4)$ & $0.659(a)$ \\
\hline $4-6$ & $1(3.4)$ & $5(8.6)$ & \\
\hline \multicolumn{4}{|c|}{ Eliminación de excretas } \\
\hline Baño & $28(96.6)$ & $53(91.4)$ & $0.659(a)$ \\
\hline Letrina & $1(3.4)$ & $5(8.6)$ & \\
\hline \multicolumn{4}{|c|}{ Piso de vivienda } \\
\hline Tierra & $9(31.0)$ & $22(37.9)$ & $0.527(\mu)$ \\
\hline Cemento & $20(69.0)$ & $36(62.1)$ & \\
\hline \multicolumn{4}{|c|}{ Animales domésticos en casa } \\
\hline Sí & $16(55.2)$ & $32(55.2)$ & $1.000(\mu)$ \\
\hline No & $13(44.8)$ & $26(44.8)$ & \\
\hline
\end{tabular}




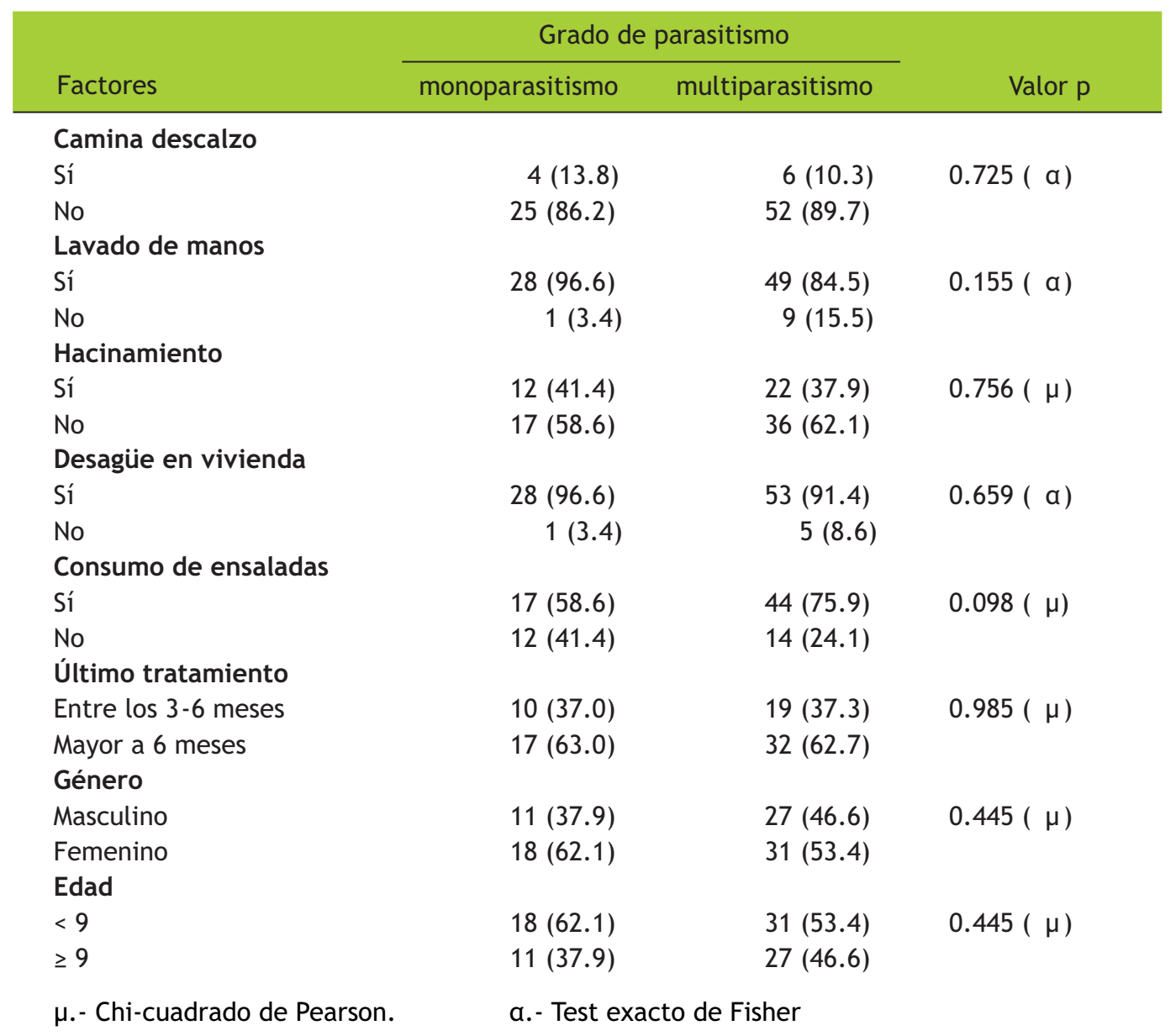

\section{DISCUSIÓN}

El presente estudio mostró alta prevalencia parasitaria $90.6 \%$ en los niños preescolares y escolares participantes, siendo mayor a otros reportes dados en esta parte de la sierra como el de Rúa y col. en Llama $80.7 \%$, Cieza y col. en Chota $74.0 \%$, Rivera y col. en el distrito de Cajamarca $48.9 \%$, y muy parecido a lo encontrado en Puno $91.2 \%$, Arequipa $92.68 \%$ e incluso Trujillo $91.3 \%$ aunque menor a lo hallado por Cholán y col. $100 \%$. Esto indicaría alta presencia de enteroparásitos en niños preescolares y escolares que son atendidos en el centro médico EsSalud del distrito de Celendín. También predominó el multiparasitismo, es decir, la mayoría de muestras positivas tenían más de una forma parasitaria, contrario al estudio de Rivera y col. donde fue el monoparasitismo que predominó ${ }^{(11,13,14,15,17,19,20)}$.

También se evidenció mayor presencia de protozoos frente a helmintos igual que en otros estudios realizados en costa y sierra, siendo muchos los factores que lo ocasionan, como las condiciones climáticas, la calidad del suelo en la sierra, la humedad, etc. Que impiden el desarrollo de formas infectantes. Cabe mencionar que también el método de concentración usado para este estudio habría influenciado en su baja presencia ${ }^{(1,11,15,16,17,22,23,24)}$.

Blastocystis hominis fue hallado en un $81.2 \%$, una prevalencia alta en relación a otros estudios realizados en el departamento de Cajamarca. Si bien es cierto que aún está en debate su patogenicidad, su potencial zoonótico, su alta prevalencia en países sub-desarrollados, el ser un nuevo indicador epidemiológico de fecalismo y servir como marcador para la presencia de otro parásito, lo hacen muy importante. El no haber usado preservantes para las muestras habría generado pérdida pues es un anaerobio estricto y sensible al clima, siendo el clima de Celendín, frio y caluroso ${ }^{(6,10,13,16,17,19,20,25,26)}$.

Entamoeba coli fue el segundo parásito hallado en el estudio con mayor porcentaje $35.4 \%$, aunque menor a lo encontrado por Rivera y col. en las guarderías de la 
zona periurbana y rural del distrito de Cajamarca 47.8\%. Otras zonas con alta presencia de Entamoeba coli son Puno $78 \%$ y Arequipa $72.68 \%$. Este protozoo comensal está presente en climas cálidos y húmedos, también sirve como un indicador fecal del agua y de deficiencias en los sistemas de alcantarillados. Se le ha encontrado también en las heces de algunos animales que son portadores $s^{(6,11,14,15,27)}$.

lodamoeba butschlii, Endolimax nana y Chilomastix mesnili son parásitos comensales que también fueron hallados pero en menor proporción. Estos protozoos también están presentes cuando las condiciones sanitarias son inadecuadas y hay una deficiente política en salud ${ }^{(6,16)}$.

Giardia lamblia tuvo una baja prevalencia tanto global como la prevalencia encontrada en preescolares de nuestro estudio (Tabla 2), muy diferente a lo presentado por Rivera $39.1 \%$ y Cholán $47.0 \%$ en niños de guarderías y jardines aunque semejante a lo obtenido en Llama 9.1\%. Altas prevalencias a Giardia lamblia están presentes en niños en edad preescolar y que aun dependen de sus padres para realizar su higiene personal. La deficiencia en dicha higiene y su transmisión por el agua de consumo, pese a estar clorada, son uno de los factores que aumentan su presencia ${ }^{(2,7,14,17,20)}$.

Como se mencionó anteriormente, las condiciones para que los helmintos proliferen en la sierra no son adecuadas, es por ello que en solo un caso de los niños muestreados se encontró Ascaris lumbricoides, aun así su baja presencia encontrada en nuestro estudio, podría deberse también a la poca sensibilidad del método de concentración usado. Estudios en otras zonas de Cajamarca, usando diferentes métodos de concentración y de flotación, encontraron prevalencias a Ascaris lumbricoides del $18.8 \%, 21.7 \%$ e incluso $47.0 \%$ así como se encontraron otros tipos de helmintos ${ }^{(14,19,20)}$.

Enterobius vermicularis fue el único helminto que tuvo mayor prevalencia en el estudio, aunque menor a los reportes de la costa, donde se halla presente hasta en un $66 \%$ de las muestras parasitadas. Algunas causas que influyeron en su poca presencia en el presente estudio fueron: olvido de traer la muestra, inadecuada toma de muestra por parte del familiar y traer láminas rotas de difícil lectura ${ }^{(1,9,12,13)}$.

Muy aparte de los parásitos encontrados, se pudo hallar en un $20.8 \%(20 / 96)$ de los niños, unas estructuras con forma globular grasosa (Figura 1) y que actualmente está generando controversia por el personal del laboratorio clínico de nuestro país, pues algunos lo están considerando como simple artefacto o restos vegetales mientras que otros no. Lo cierto es que el investigador Francisco Tirado Santamaría, catedrático de Parasitología en la Universidad Industrial de Santander (Colombia), ha venido estudiando estas estructuras concluyendo que se trata de un nuevo tipo de protozoo al que lo ha denominado como Urbanorum spp. Si bien es cierto que dicho parásito aún se encuentra bajo estudio y no cuenta hasta el momento con referencias bibliográficas, su importancia médica a futuro como nuevo parásito patógeno está siendo evaluada ${ }^{(28)}$.

Figura 1. Examen directo con lugol parasitológico donde se observó estructuras sugerentes a parásito Urbanorum spp. (Aumento 400x)

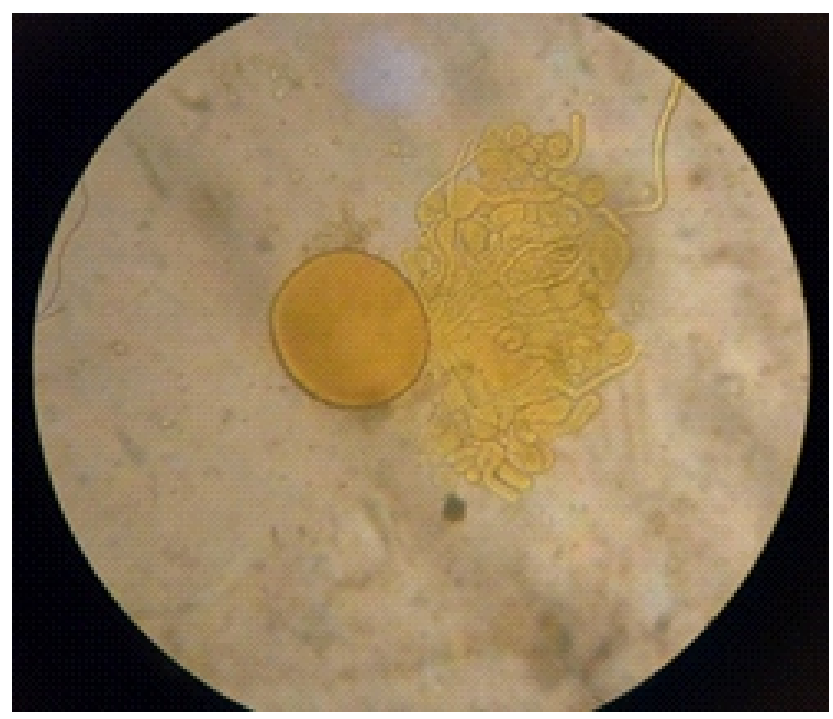

De los datos sociodemográficos e interrelación personal obtenidos, la gran mayoría de niños provenía de la zona urbana $85.4 \%$ donde supuestamente existe una mejora en los servicios de alcantarillado, agua y desagüe, una política de higiene promovida por instituciones de salud así como el gozo de buena salud en contraste a los niños que habitan la zona rural, evidenciándose en variables como el consumo de agua potable $89.6 \%$, el lavado de manos $85.5 \%$, el empleo de desagües para eliminar las excretas $93.8 \%$ y el uso del baño $93.8 \%$. Otras variables evaluadas fueron el hacinamiento, el cual estuvo ausente en el $62.5 \%$, que podría haber disminuido el contagio parasitario entre niños que habitan un mismo cuarto, por otra parte el $97.9 \%$ de los niños tuvieron lactancia materna exclusiva, es decir hubo protección inmunológica por parte de la madre hacia el niño contra agentes parasitarios. También buena parte habían recibido tratamiento. Si bien estos resultados 
harían pensar en una baja presencia parasitaria, fue totalmente contradictorio a la alta prevalencia encontrada, resultado que si estaría relacionado con otras variables como el alto consumo de ensaladas $70.8 \%$, la cantidad de miembros o hijos que hay en una familia, la presencia de animales domésticos en casa 57.3\%. La deficiencia de algunas variables mencionadas primeramente como la sospecha del maltratamiento del agua potable, donde se ha visto que los quistes han generado resistencia a su potabilización, así como una mala higiene también aumentaría aún más dicha presencia ${ }^{(7,10,29)}$.

Por otra parte el alto porcentaje de niños que no andaban descalzos $87.5 \%$ así como la baja presencia de pisos de tierra en las viviendas $35.4 \%$, podrían haber disminuido mucho más la presencia de helmintos en el estudio.

La examinación clínico médica obtuvo síntomas asociados a parasitosis en niños, tal como el dolor abdominal, diarrea, estreñimiento, prurito anal e inapetencia. Típicos para esta clase de infecciones ${ }^{(8,9,17,22)}$.

El nivel de educación fue el único que tuvo dependencia con el grado de parasitismo $(p=0.017)$, esto se debería en aquellos niños mayores que ya se encuentran en el nivel escolar (sea primaria o secundaria) y que se les presta menos atención a su higiene personal por parte de los padres, quienes están más pendientes o dedicados al aseo del menor hijo.

En conclusión, se encontró alta prevalencia de parasitosis en niños en edad preescolar y escolar del distrito de Celendín que fueron atendidos en el centro médico EsSalud, siendo Blastocystis hominis el parásito más predominante. Este trabajo es uno de los primeros que se realiza en el distrito de Celendín, que pese a ser una zona urbanizada, aún persiste la deficiencia a nivel sanitario, lo que genera la presencia de parásitos.

Agradecimiento: A Gabriel Alcántara De La Rosa, doctor en medicina, por permitir la realización del presente trabajo dentro del Centro Médico EsSalud Celendín, Cajamarca.

\section{REFERENCIAS BIBLIOGRÁFICAS}

1. Jiménez J, Vergel K, Velásquez García-Sayán M, Vega F, Uscata $\mathrm{R}$, Romero $\mathrm{S}$ et al. Parasitosis en niños en edad escolar: relación con el grado de nutrición y aprendizaje. Revista Horizonte Médico 2011; 11(2): 65-69.

2. Alvarado Lázaro L, Romero Sánchez Y M. Nivel de conocimiento y práctica de conductas promotoras en docentes de nivel inicial para la prevención de parasitosis intestinal. Distrito Florencia de Mora, 2013. [Tesis de pregrado]. Trujillo. Facultad de ciencias de la salud. Escuela profesional de enfermería. Universidad privada Antenor Orrego; 2013.

3. Sánchez de la Barquera-Ramos M A, Miramontes-Zapata M. Parasitosis intestinales en 14 comunidades rurales del altiplano de México. Rev Mex Patol Clin 2011; 58 (1): 16-25.

4. Al Rumhein F, Sánchez J, Requena I, Blanco $Y$, Devera R. Parasitosis intestinales en escolares: relación entre su prevalencia en heces y en el lecho subungueal. Rev Biomed 2005; 16:227-237.

5. Suca Inga M, Valle Tiza C, Gonzales Aylaz M, Diaz Lizana J, Samaniego J J, Milian Jímenez W et al. Parasitosis intestinal en niños del PRONOEI módulo 05 Manzanilla, Lima-Perú. Rev Med Rebagliati 2013: 5(5) 12-14.

6. Juárez M M, Rajal V B. Parasitosis intestinales en Argentina: principales agentes causales encontrados en la población y en el ambiente. Rev Argent Microbiol. 2013; 45(3):191-204.

7. Gamboa M I, Zonta L, Navone G T. Parasitosis intestinales y pobreza: la vulnerabilidad de los más carenciados en la Argentina de un mundo globalizado. J Selva Andina Res Soc 2010; 1(1): 23-36.

8. İpek Östan, Ali A Kilimcioğlu, Nogay Girginkardesler, Beyhan C Özyurt, M Emin Limoncu and Ülgen Z Ok. Health inequities: lower socio-economic conditions and higher incidences of intestinal parasites. BMC Public Health 2007, 7:342.

9. Teresa Navone G, Gamboa M I, Oyhenart E E, Orden A V. Parasitosis intestinales en poblaciones Mbyá-Guaraní de la provincia de Misiones, Argentina: aspectos epidemiológicos y nutricionales. Cad Saúde Pública, Rio de Janeiro 2006, 22(5):1089-1100.

10. Gamboa M I, Navone G T, Kozubsky L, Costas M E, Cardozo M, Magistrello P. Protozoos intestinales en un asentamiento precario: manifestaciones clínicas y ambiente. Acta Bioquím Clín Latinoam 2009; 43 (2): 213-8.

11. Casquina G L, Martínez B E. Prevalencia y epidemiología del parasitismo intestinal en escolares de nivel primario de Pucchún, Camaná, Arequipa, Perú, 2006. Neotropical Helminthology 2011; 5 (2): 247-255.

12. Ubillus G, Ascarrus A, Soto C, Medina J, Falconí R, Franco J et al. Enteroparasitosis y estado nutricional en niños menores de 4 años de wawa-wasi de Pamplona Alta San Juan de Miraflores Lima-Perú-2006. Revista Horizonte Médico. 2008; 8 (2): 36-40.

13. Navarro Caballero M M. Prevalencia de parasitosis intestinal y factores epidemiológicos asociados en escolares del asentamiento humano Aurora Díaz de Salaverry-Trujillo [Tesis de pregrado]. Trujillo. Biblioteca digital. Oficina de sistemas e informática. Universidad Nacional de Trujillo. 2013.

14. Rivera Jacinto M, López Orbegoso J, Rodríguez Ulloa C. Enteroparasitosis Infantil en Guarderías de la zona rural de Cajamarca. Rev Peru Med Exp Salud Pública. 2008; 25(4): 344-49.

15. Maco Flores V, Marcos Raymundo L A, Terashima Iwashita A, Samalvides Cuba F, Gotuzzo Herencia E. Distribución de la enteroparasitosis en el altiplano peruano: estudio en 6 comunidades rurales del departamento de Puno, Perú. Rev. Gastroenterol. 2002; 22 (4): 304-309.

16. Pajuelo Camacho G, Lujan Roca D, Paredes Pérez B. Estudio de enteroparásitos en el hospital de Emergencias Pediátricas, Lima-Perú. Rev Med Hered. 2005; 16 (3): 178-183. 
17. Oliver R, Romero G, Romaní F. Prevalencia de parasitosis intestinal en escolares de una institución educativa de un distrito de la sierra peruana. Rev Peru epidemiol. 2010; 14 (2): 161-165.

18. Rodríguez Ulloa C, Rivera Jacinto M, Saucedo Duran E, Rojas Huamán Y, Valdivia Meléndez N, Cabanillas Vásquez Q, Blanco Burga $\mathrm{H}$. Parasitosis intestinales y factores socio-sanitarios en niños del área rural del distrito de Los Baños del Inca, Cajamarca-Perú. Rev Med Hered. 2010; 21: 107-108.

19. Cieza D, Hurtado M. Universidad Nacional de Cajamarca. Incidencia de parasitismo intestinal en alumnos del C.E. 10385 "Santa Rafaela María" de Chota, Rev Caxamarca. 2003; 11(3):9-16.

20. Cholán S, Boyd M, Guerra R. Prevalencia y distribución de enteroparásitos en aldeas Hogar de Dios "Ayuda a tu prójimo" de la ciudad de Cajamarca. Febrero-Abril 2002. Rev Peru Med Exp Salud Pública 2002; 19 (Supl): 24-25.

21. Instituto Nacional de Salud. Manual de procedimientos de laboratorio para el diagnóstico de los parásitos intestinales del hombre. INS-serie de normas técnicas № 37 Lima-2003.

22. Maco Flores V, Marcos Raymundo L A, Terashima Iwashita A, Samalvides Cuba F, Gotuzzo Herencia E. Prevalencia de parasitosis intestinal en niños del valle del Mantaro, Jauja, Perú. Rev Med Hered. 2002; 13: 85-89.

23. Iannacone J, Benites M J, Chirinos L. Prevalencia de infección por parásitos intestinales en escolares de primaria de Santiago de Surco, Lima, Perú. Parasitol Latinoam. 2006; 61: 54-62.

24. Cabrera M, Verástegui M, Cabrera R. Prevalencia de enteroparasitosis en una comunidad altoandina de la provincia de Víctor Fajardo, Ayacucho, Perú. Rev Gastroenterol Perú [en línea] Abril-Junio 2005 [Fecha de acceso: 10 Enero 2016]; 25(2). URL disponible en: http://www.scielo.org.pe/ scielo.php? script = sci_arttext\&pid=S1022. 51292005000200003

25. Méndez Bustelo M A, Do Muiño joga M, Garabal Sánchez S, Ben López E, Llovo Taboada J S, Blastocystis hominis, una gran desconocido. Rev Pediatr Aten Primaria. 2015; 17: 39-44.
26. Cañete Villafranca R, Rodríguez Jiménez P. Infección por Blastocystis sp.: revisión de la literatura. Rev Méd Electrón [en línea] Setiembre-Octubre 2012. [Fecha de acceso: 21 Enero 2016]; 34(5). URL disponible en: http://www.revmatanzas. sld.cu/revista\%20medica / a no\%202012/vol5\%202012/ tema05.htm

27. Altamirano F, López R, Puray N. Enteroparásitos con potencial Zoonótico en pacientes pediátricos del hospital de Andahuaylas-Apurímac. Salud tecnol vet. 2014; 2: 14-19.

28. Publicando viral [homepage en Internet]. Colombia: Publicando viral; c2013 [actualizada 23 de Octubre del 2013; consultado 25 Enero 2016]. Disponible en: http://publicandoviral. blogspot.pe/2013/10/nuevoparasito-intestinal-descubierto.html

29. Quihui Cota L, Lugo Flores C M, Morales Yocupicio T E, Cubillas Rodríguez M J, Abril Valdez E M, Román Pérez R, et al. Parasitosis intestinales en escolares urbanos, suburbanos y rurales del noroeste de México. Biotecnia. 2014; 16 (2):15-20.

Fuentes de Financiamiento

Este artículo ha sido financiado por los autores.

Conflictos de interés

Los autores declaran no tener ningún conflicto de interés.

Correspondencia:

Jimmy Rinaldo Morales Del Pino

Dirección: Jr. Río putumayo 3235, Urb. Canto Rey-San Juan de Lurigancho-Lima

Teléfono: (01)387-9018

Correo electrónico: martirio3452@hotmail.com

Recibido: 21 de abril de 2016 Aprobado: 04 de julio de 2016 